\title{
BIOQ-BYWORD
}

\section{An Evaluative Research of Preventive Effect of Antenatal Kegel Exercise on Postpartum Stress Urinary Incontinence}

\author{
Xu Jingjing, Zhang Qing,Cao Shuyan
}

Obstetrical Department, Nanjing General Hospital of Nanjing Military Command, Nanjing, 210000, China

\section{ARTICLE INFO}

Article history:

Published online: $30^{\text {th }}$ Sept, 2017

\section{Key words: \\ Antenatal Kegel Exercise \\ Postpartum Stress Urinary \\ Incontinence}

Motivational Interviewing

\begin{abstract}
Objective: The aim of this study was to evaluate the effects and effectiveness of antenatal Kegel exercise on prevention of postpartum stress urinary incontinence. Methods: Totally 92 pregnant women who had Perinatal care card in Nanjing General Hospital of Nanjing Military Command were randomly divided into two groups. The pregnant women in the control group received routine health education, while the pregnant women who has 37 weeks of pregnancy in the experimental group received antenatal Kegel exercise and motivational interviewing intervention. The experimental group exercise 150-200 times every day. The incidence of SUI were measured after puerperae deliveried 42 days and 3 months. Results: The incidence of SUI was much more lower in the experimental group; the significant difference was found between the two groups. Conclusion: Antenatal Kegel exercise is more effective than routine health education on the prevention of postpartum stress urinary incontinence.
\end{abstract}

\section{Introduction}

Stress Urinary Incontinence (SUI) is now one of the five most common chronic diseases endangering women health ahead of hypertension, depression and diabetes according to some surveys conducted in developed countries. Its occurrence is often related to genitourinary organ prolapse and pelvic floor injury caused by pregnancy or delivery whose medical costs have outnumbered coronary heart disease, osteoporosis and breast cancer, etc. Kegel exercise is a kind of active pelvic floor exercises working on pubococcygeus which is now recognized as a simple, painless and effective method for preventing and treating Postpartum Urine Incontinence in constant practice in the long run. Extensive evidence-based medicine researches have been conducted on Kegel exercise in China these years with the number of related literature within 5 years reaching 49 , achieving a great effect. However, essays on antepartum Kegel exercise are only up to 4 . Thus, this work applies Kegel exercise into antepartum pelvic floor exercise so as to monitor and assess the effect and feasibility of Kegel exercise for preventing postpartum SUI.

\section{Research Object}

The research objects of this work were chosen from pregnant women who were recorded in perinatal care files of our hospital since the February of 2014. Choice criteria: (1) those who have no urine incontinence during pregnancy; (2) those whose age are from 26 to 30 and 37 weeks of gestation; (3) those whose neonate will be less than $4 \mathrm{~kg}$ in weight and consistent with actual gestation age in size; (4) those who are pregnant with single fetus for the first time; (5)those who have normal BMI $(19 \leq \mathrm{BMI}<24)$; 6 6 those who have no complication or cephalopelvic disproportion and choose natural childbirth. Exclusion criteria: (1) those who get hemorrhoids; (2) those who have no history of pelvic operation; (3) those who are not able to choose natural childbirth since force majeure; (4) those who reject prenatal exercises. Random 
sampling method was conducted in the excluding process, then all chosen objects were labeled and divided into the experienmental group of 44 objects and the control group of 48 objects. 8 objects were excluded due to rejecting prenatal exercises or losing contact, etc. Two groups showed no significant difference when comparing age, weeks of gestation, education level and neonate weight $(\mathrm{P}>0.05)$.

\section{Method}

\subsection{Intervention Method}

The two groups were offered with corresponding intervention methods for 4 months in a row. The pregnant women were provided with the first face-to-face intervention at the 36th week of gestation. Basic information were gathered before the first intervention, then the instruction of prenatal Kegel exercise and routine health education were given on weekly basis till delivery, while the control group were given routine health education. All nurses undertaking the work have acquired qualifications beyond chief nurse and plentiful clinic experience and have accepted instructions of Kegel exercise and MI technique training.

\subsubsection{Experimental Group}

The experimental group accepted Kegel exercise at the end of 37th week of gestation for 150-200 reps on a daily basis at self-selected time until delivery. We established MI intervention plan according to different object and conducted one-on-one intervention based on the plan. During the first intervention, the interviewer communicated with patients via individualized interacting skills in order to build up a relationship of trust as well as to get informed with existed questions, mental situation, daily customs and ways of working, etc. The Kegel exercise instruction was given through communication in person before relevant assessment: The pregnant should take the horizontal position with a soft pillow under her head; she should then bend her knees and spread the legs slightly, simulating urination (or defecation). Tighten and relax pelvic floor muscle; breathe in for 3-5 seconds and relax when contracting anus; remind the pregnant to keep thighs and abdominals relaxed when contracting anus, vagina and urethra. Help the pregnant practice repeatedly until she completely masters it. The interventions were conducted on weekly basis during antepartum inspection which focuses on observing the compliance of the pregnant and evaluating the feasibility of household conduction. An interactive discussion should be held to know how well their work-out is and to provide targeted guidance for ensuring the therapeutic effect.

\subsubsection{Control Group}

The control group was given routine health education.

\subsection{Assessment Method}

The incidence of SUI was measured when the puerperas came back for re-inspection 42 days after delivery and by calling back 3 months after delivery. The simplified form of ICI questionnaire (ICI-Q-SF) was used as the assessment method: Please answer the following questions after thinking about how well you have been: (1) How often do you leak urine? (2)We would like to know how much urine you think leaks. (3) Overall, how much does leaking urine interfere with your everyday life? The incidence of SUI is assessed by the following terms: (1) $1-5$ points according to the frequency of weekly urine leak; (2) 2-6 points according to the urine-soaked area on the urine mat; (3) 1-10 according to the seriousness of the impact urine leak have on personal life. The sum of all points is the total score of ICI-Q-SF. The more the score is, the more serious SUI is: mild SUI when the score is less than or equal 6; medium SUI when the score is higher than 7 and less than 12; severe SUI when the score is higher than 13 .

\subsection{Statistic Method}

SPSS19.0 statistic software was adapted for data processing. The counting index was represented by percentage numbers and examined by chi-square test where $p<0.05$ stands for the fact that differences are statistically significant.

\section{Result}

SUI Occurrence Comparison (Table 1, Table 2) The data suggests that the incidence of the experimental group is obviously lower than the control group, in which the difference is statistically significant $(\mathrm{P}<0$. 05). According to the severity of SUI, the control group is more severe than the experimental group, in which the difference is statistically significant $(\mathrm{P}<0.05)$.

\section{Discussion}

\subsection{SUI is one of the most important diseases affecting women's life quality}

Natural childbirth oppresses and over-stretches the muscle, fascia and ligament of pelvic floor support structure, leading to functional disorders due to some irreversible changes to the structures above both in form and function such as gatism and pelvic floor organ prolapse, etc. It is reported that $25 \%-55 \%$ of the pregnant suffer from urinary incontinence during pregnancy, $3.7 \%-19 \%$ of which experience the first occurrence after natural delivery. $34.3 \%$ of the women suffer from different degree of SUI 3 months after delivery. However, natural childbirth is not the only factor that causes pelvic floor function disorder. The pregnancy itself does harm to the pelvic floor since the pregnancy makes pelvic muscle bear abdominal pressure and weights of pelvic organs. Coupled with the increase of womb weights, pelvic muscle suffers from constant pressure, which results in 
Table 1 Compared with the incidence of SUI after postpartum 42th day between the two group of puerperae (\%)

\begin{tabular}{ccccccc}
\hline \multirow{2}{*}{ Group } & \multirow{2}{*}{ Objects } & \multicolumn{4}{c}{ SUI severity } & \multirow{2}{*}{ Indicence (\%) } \\
\cline { 3 - 6 } & & None & Mild & Medium & Severe & \\
\hline Experimental group & 44 & 44 & 0 & 0 & 0 & 0 \\
Control group & 48 & 41 & 5 & 2 & 0 & 14.6 \\
\hline
\end{tabular}

Note:Comparing the incidence and the severity, $\mathrm{P}<0.05$

Table 2 Compared with the incidence of SUI after postpartum 3rd month between the two group of puerperae (\%)

\begin{tabular}{ccccccc}
\hline \multirow{2}{*}{ Group } & \multirow{2}{*}{ Objects } & \multicolumn{4}{c}{ SUI severity } & \multirow{2}{*}{ Indicence (\%) } \\
\cline { 3 - 6 } & & None & Mild & Medium & Severe & \\
\hline Experimental group & 44 & 42 & 2 & 0 & 0 & 4.55 \\
Control group & 48 & 37 & 9 & 1 & 1 & 22.9 \\
\hline
\end{tabular}

Note: Comparing the incidence and the severity, $\mathrm{P}<0.05$

Other researches demonstrate that $80 \%$ of women experienced changes in nerve conduction with pelvic floor tissue after the first natural childbirth through electrophysiological experiments. Other obstetric factors such as multiple childbirths, prolonged second stage of delivery, overweight fetus or oversized fetus head or perineotomy could also cause partial denervation effect for pelvic floor muscle and urethral striated muscle, increasing the possibility of SUI occurrence. Researches from Wilson and Morkved Et al. suggests that UI is a common issue 3 months after delivery, affecting about $40 \%$ of the puerpera. Although the symptoms of UI disappear after 6 months for most of women, it is shown by this work that the incidence increased after 3 months than after 42 days. According to the data, the SUI incidence of the control group rose from $14.6 \%$ after 42 days to $22.9 \%$ after 3 months; and that of the experimental group increased from none to $4.55 \%$. Nevertheless, studies by Dolan Et al. proved that the risk of SUI within 15 years would double if SUI happens during the first pregnancy, which should arouse the attention from obstetric staff on preventing postpartum UI.

\subsection{The feasibility of Kegel exercise}

Evidences indicate that the pregnant women could adapt mild or moderate exercises which Kegel exercise belongs to. In Physical Activity and Exercise During Pregnancy and the Postpartum Period published by ACOG in 2002, Kegel exercise is recognized as a means of work-out during pregnancy since its intensity is weaker than Prenatal Yoga, and its moves are easy to master without any supplementary equipment or constrains of time, location or posture. The exercise can be conducted while lying, sitting or standing, owning a relatively good compliance and feasibility. Especially, constantly conducting Kegel pelvic floor muscle function exercise can prevent SUI effectively.

\subsection{Strengthen the education of prenatal Kegel exercise}

Through the surveys made by pregnant women schools on awareness of SUI we know that only $20 \%$ of the pregnant women learn about UI briefly, showing an enormous lack of relevant knowledge of postpartum urinary incontinence among the pregnant women and purepera. Clinic cases also point out that factors such as breast-feeding, wound pain and

insufficient exercise reps interrupt and influence the constant conduction of the exercise, which makes the lack of persistence a major cause of an uncertain curative effect. What is more, an unwilling attitude from the family is also one of the major factors, suggesting the lack of relevant knowledge of prenatal exercises among the public. Thus, we should inform the pregnant women of the causes of SUI, multiple ways for prevention and the importance of Kegel exercise by various forms of health education such as billboards, health prescriptions, lectures and pregnant women schools, leading them to conduct pelvic floor exercise actively in order to decrease the incidence of

postpartum urinary incontinence.

\section{Conclusion}

This research shows that Kegel exercise has an obvious effect on preventing postpartum SUI. Also, the MI method should be adapted to raise the compliance of 
pregnant women, letting them accept and master Kegel exercise. However, there is no integrated standard on the time choice and reps of pelvic floor exercise. And various forms of prenatal exercise such as Prenatal Yoga need further research due to many constrains on sites and devices, etc.

\section{References}

[1] Diem I-IP, I $\infty \infty^{2}$ rone V. Levator trauma after vaginal delivery[J] . Obstet Gynecol. 2005, 106(4):707-712 .

[2] Yang Junling, Xie Li, Zhang Peilian. Effect of postpartum pelvic floor muscle training to prevent urinary incontinence [J]. CHINESE JOURNAL OF NURSING, 2008,43(5):436-437.

[3] Wang Xin, Li Guiyou, Deng Meilian. Effect of persistent guidance of pelvic floor muscle training on the delivery outcome and pelvic muscle strength [J]. Chinese Journal of Nursing,2013,48(4): 308-310.

[4] Da Peng, Wang Shanmi. Early postpartum urine loss and pelvic floor muscle training [J]. Chinese Journal of Clinical Obstetrics and Gynecology, 2004,5(1): 66-67.

[5]Filocamo MT,Li Marzi V,Del Popolo G, et al. Effectiveness of early pelvic floor rehabilitation treatment for post-prostatectomy incontinence[J].European Urology,2005,48(5):734-738.

[6]Zmrhal J, Horcica L. The changes of electromyographic activity of the pelvic floor after the delivery[J].Zentralbl Gyrmekol, 2001, 123(11): 619-621. [7] Wihun PD, Herbison RM, Herbison GP. Obstetric practice and the prevalence of urinary incontinence three months after delivery[J] . Br J Obstet Gyunecol, 1996, 103(2): 154-161.

[8] Wilson PD, Herbison RM, Herbison GP. Obstetric practice and the prevalence of urinary incontinence three months after delivery[J] . Br J Obstet Gynaecol, 1996, 103(2): 154-161.

[9]Morkved S, Bo K. Prevalence of urinary incontinence during pregnancy and postpartum[J]. Urogynecol J Pelvic Floor

Dysfunct, 1999, 10(6):394-398.

[10]Thorp JM, Norton PA , Wall LI., et al, Urinary incontinence in pregnancy and the puerperium : a prospective study[J]. AM J Obstet Gynecol. 1999, 181(2): $266-273$.

[11]Dolan LM, Hosker GL . Mallett VT., et al. Stress division and incontinence and pelvic floor neurophysiology 15 years after the first delivery[J]. Br J Obstet Gynaecol, 2003, 110: 1107-1114.

[12] Tan Minjuan, Li Jun. Research progress of the physical exercises and sports pattern during pregnancy $[\mathrm{J}]$. CHINESE JOURNAL OF NURSING,2011,(46)2:199-201. 